\title{
Effect of a nanoparticle on the optical properties of a photonic crystal cavity: theory and experiment
}

\author{
Toeno van der Sar, ${ }^{1, *}$ Jenna Hagemeier, ${ }^{2}$ Wolfgang Pfaff, ${ }^{1}$ Erwin Heeres, ${ }^{3}$ Susanna Thon,${ }^{2,6}$ Hyochul Kim, ${ }^{2,7}$ \\ Pierre Petroff, ${ }^{4,5}$ Oosterkamp Tjerk, ${ }^{3}$ Dirk Bouwmeester, ${ }^{2,3}$ and Ronald Hanson ${ }^{1}$ \\ ${ }^{1}$ Kavli Institute of Nanoscience, Delft University of Technology, P.O. Box 5046, 2600 GA Delft, The Netherlands \\ ${ }^{2}$ Department of Physics, University of California Santa Barbara, Santa Barbara, California 93106, USA \\ ${ }^{3}$ Leiden Institute of Physics, Leiden University, Niels Bohrweg 2, 2333 CA Leiden, The Netherlands \\ ${ }^{4}$ Department of Materials, University of California Santa Barbara, Santa Barbara, California 93106, USA \\ ${ }^{5}$ Department of ECE, University of California Santa Barbara, Santa Barbara, California 93106, USA \\ ${ }^{6}$ Current address: Maitland St. Suite 401, Toronto, ON M4Y1E5, Canada \\ ${ }^{7}$ Current address: Department of ECE, IREAP, University of Maryland, College Park, Maryland 20742, USA \\ ${ }^{*}$ Corresponding author: t.vandersar@tudelft.nl
}

Received October 6, 2011; revised January 2, 2012; accepted January 4, 2012;

posted January 5, 2012 (Doc. ID 156138); published March 20, 2012

Single quantum emitters can be coupled to photonic crystal (PC) cavities by placing their host nanoparticles into the cavity field. We describe fabrication, characterization, and tuning of gallium-phosphide PC cavities that resonate in the visible, and simulations and measurements of the effect of a nanoparticle on the optical properties of these cavities. Simulations show that introducing a $50 \mathrm{~nm}(100 \mathrm{~nm})$ sized nanoparticle into S1 and L3-type cavities, with original quality factors of $18 \cdot 10^{3}$ and $73 \cdot 10^{3}$, respectively, reduces the quality factor by $<10 \%(\sim 50 \%)$. Furthermore, simulations indicate that an emitter embedded in a $50 \mathrm{~nm}(100 \mathrm{~nm})$ sized nanoparticle can be coupled 3.5 (9) times more effectively to an S1 cavity than to an L3 cavity. We employ a nanopositioning technique to position individual, $50 \mathrm{~nm}$ sized nanocrystals into S1 cavities, and find that the quality factors are reduced by a factor of $0.9 \pm 0.1$ from the original values of order $10^{3}$. (c) 2012 Optical Society of America

OCIS codes: $\quad 050.5298,350.4238,270.0270$.

\section{INTRODUCTION}

Photonic crystal (PC) cavities can confine light to a volume smaller than the wavelength with a high quality factor, making them prime candidates for applications in quantum electrodynamics (QED) [1,2]. The strongly enhanced vacuum fluctuations in PC cavities can be used to control and engineer the emission properties of single quantum emitters, enabling, e.g., efficient generation of nonclassical light and the exploration of cavity QED into the strong-coupling regime [3, $\underline{4}]$.

A primary challenge in deterministically coupling a single quantum emitter to a $\mathrm{PC}$ cavity is to position the emitter into the nanometer-sized optical mode, requiring precise spatial control over either the emitter or the cavity. While single, selfassembled quantum dots can be naturally embedded in the PC material during substrate growth, after which a PC cavity can be fabricated around a specific dot using precise lithographic techniques [5,6], other types of single quantum emitters generally require a different approach $[\underline{7}, \underline{8}]$.

Recently, the development of precise positioning techniques has started a new field of experiments where quantum emitters contained in nanometer-sized particles can be deterministically coupled to PC cavities [9]. In particular, coupling of single nitrogen-vacancy (NV) centers embedded in diamond nanocrystals to a PC cavity has recently been demonstrated by a number of groups $[\underline{8}, \underline{10}, \underline{11}]$.

Studies of the interaction between a single quantum emitter and a PC cavity, such as those described in [5-11], commonly make use of L3 and S1 cavities (Fig. 1), as simulations indicate that these can have high quality factors. The high quality factor, essential for realizing strong interaction between emitter and cavity, is a result of careful structural design and can therefore be expected to degrade upon the introduction of a small particle into the cavity mode [12]. Here, we study the effect of the introduction of a nanoparticle into gallium-phosphide (GaP) PC cavities by simulations and measurements. We first describe simulations of the effect of introducing a nanoparticle into L3 and S1 cavities on the cavity quality factor and resonance wavelength. We then turn to experiment, starting with a description of the fabrication, characterization, and tuning of PC cavities, followed by a description of measurements of the quality factor of six different PC S1 cavities before and after the introduction of individual $\sim 50 \mathrm{~nm}$ sized diamond nanocrystals.

\section{SIMULATIONS OF THE EFFECT OF A NANOPARTICLE ON THE OPTICAL PROPERTIES OF A PC CAVITY}

We simulated the effect of a diamond nanocrystal on the optical properties of S1 and L3 cavities using three-dimensional (3D) finite difference time domain (FDTD) simulations with grid size $12.74 \mathrm{~nm}$ in $x, y$, and $z$. Within the limitations set by this grid, a diamond nanocrystal is approximated as a spherical particle, which introduces some deviations from a true spherical shape. However, roughness on a subwavelength scale is not expected to strongly alter the profile of the calculated electromagnetic field. Furthermore, in reality, diamond nanocrystals are generally shaped irregularly. 


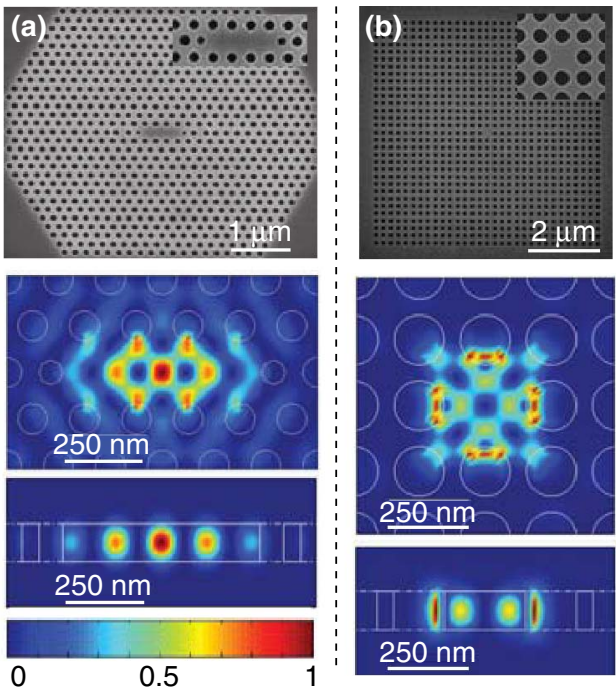

Fig. 1. (Color online) SEM pictures and simulated electric field intensity profiles of an L3 cavity (a) and an S1 cavity (b).

The refractive index of the particle was taken to be that of a diamond, $n=2.4$, and the radius of the particle was taken to be 10-100 nm, a typical size of a diamond nanocrystal containing a single NV center. The refractive index of the PC membrane was taken to be 3.4, equal to the refractive index of GaP at $600 \mathrm{~nm}$, as $\mathrm{GaP}$ is the current material of choice for coupling to single NV centers [8, 10,11$]$.

Since coupling is strongest when the emitter is placed at the location of maximum mode intensity $I_{\max }$, we perform simulations for nanocrystals placed at or near the mode maximum of a cavity. We focus on the lowest energy mode of the cavities, as it generally has the highest quality factor.

\section{A. Effect of a Nanoparticle on an S1 Cavity}

An advantage of the S1 cavity is that an external emitter can, in principle, be positioned at the location of maximum mode intensity $I_{\max }$, since $I_{\max }$ occurs inside an air hole (Fig. 1(b)). Simulations of a nanoparticle with different sizes placed at the inner edge of one of the nearest air holes surrounding the cavity, centrally in the membrane, show that $Q$, originally at $18 \cdot 10^{3}$, decreases by $<10 \%(<30 \%)$ for a nanoparticle $50 \mathrm{~nm}$ $(100 \mathrm{~nm})$ in size. For the biggest nanoparticle (nearly the size of a hole), $Q$ decreases by $\sim 65 \%$. The resonant wavelength shifts by less than $2 \mathrm{~nm}$ for nanoparticle sizes smaller than $80 \mathrm{~nm}$ (Fig. 2(a)).

Simulations of a nanoparticle $60 \mathrm{~nm}$ in size placed in the nearest air hole at various depths in the membrane show that $Q$ remains above 15000 for all placements (Fig. 2(b)). The $Q$ is highest for placement near the center of the membrane and decreases for placement near the edges, as light that is scattered by a nanoparticle at the surface is lost from the cavity.

\section{B. Effect of a Nanoparticle on an L3 Cavity}

For the L3 cavity, $I_{\max }$ occurs inside the membrane, where it is not possible to place a nanoparticle (Fig. 1(a)). Instead, it can be positioned on top of the membrane. The mode intensity at the top edge of the membrane is $\sim 0.25 \cdot I_{\max }$. Simulations with a nanoparticle of various sizes placed centrally on top of the membrane show that as the size of the nanoparticle increases, $Q$ decreases and the resonant wavelength increases (Fig. 3(a)). (a)
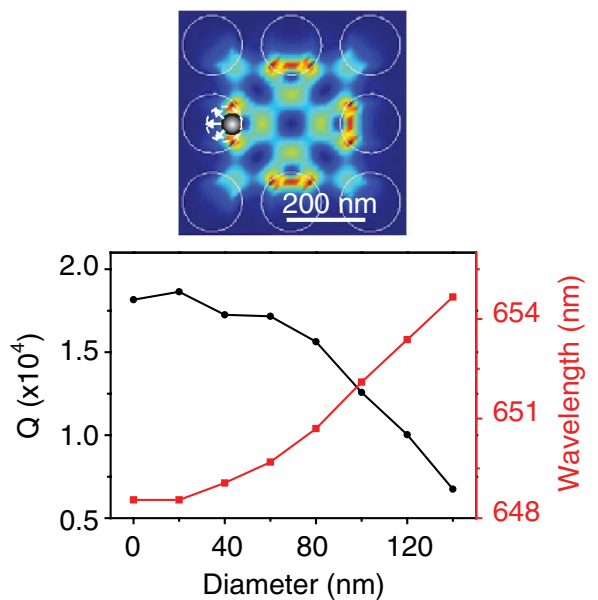

(b)

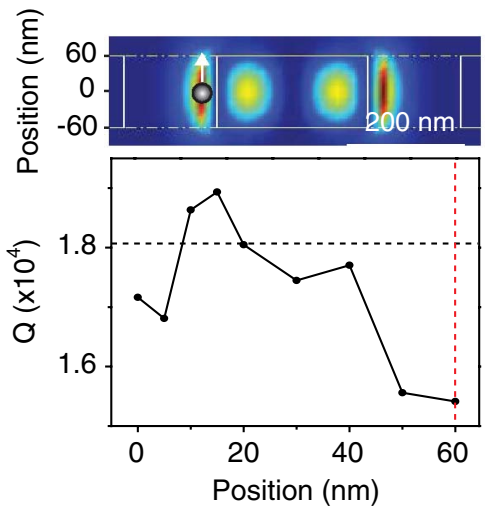

Fig. 2. (Color online) (a) Simulation of the quality factor $Q$ and the resonance wavelength as a function of the diameter of a spherical nanoparticle placed in the nearest air hole of an S1 cavity in the center of the membrane. The original structure (hole radius $r=78 \mathrm{~nm}$, lattice constant $a=205 \mathrm{~nm}$, membrane thickness $d=120 \mathrm{~nm}$ ) has a fundamental mode with wavelength $648.55 \mathrm{~nm}$, mode volume $0.56(\lambda / n)^{3}$, and $Q_{0}=1.82 \cdot 10^{4}$. The color plot indicates the position of the nanoparticle and how its size is changed. (b) Simulation of $Q$ as a function of the nanoparticle position for a spherical diamond nanoparticle (60 $\mathrm{nm}$ diameter) placed at various depths in the nearest air hole of an S1 cavity as indicated in the color plot. The red dashed line denotes the edge of the membrane. The black dashed line denotes the original $Q_{0}$.

For nanoparticles smaller than $100 \mathrm{~nm}$ in diameter, the wavelength shift is insignificant (less than $0.2 \mathrm{~nm}$ ). The $Q$, originally at $73 \cdot 10^{3}$, is reduced by $<10 \%(\sim 50 \%)$ for a $50 \mathrm{~nm}(100 \mathrm{~nm})$ sized particle.

Next, we studied the effect of a nanoparticle $100 \mathrm{~nm}$ in size located on top of the membrane of the L3 cavity as a function of its distance from the center (Fig. 3(b)). The simulations show that at the location where the potential emitter-cavity coupling is large (near the antinodes of the electric field), a nanoparticle also has the greatest detrimental effect. Light that hits the nanoparticle has a high probability to be scattered into free space since the particle is located at the surface. This is in contrast to the S1 cavity, where the optimal place to position an external emitter is inside a hole, centrally in the membrane.

\section{Simulations Summary}

In summary, our simulations indicate that an external quantum emitter can be coupled more effectively to an S1 cavity than to an L3 cavity. For both cavities, a nanoparticle does not 
strongly decrease the cavity $Q$, but the $\mathrm{S} 1$ cavity has a smaller mode volume and the nanoparticle can be placed at or very close to the mode maximum. Quantitatively, for a $50 \mathrm{~nm}$ diameter nanoparticle placed against the inner edge of a hole of an S1 cavity (as depicted in Fig. 2) with mode volume $V=0.54(\lambda / n)^{3}$, the mode intensity at the center of the particle is $I=0.58 \cdot I_{\max }$ and $Q$ is reduced from 18000 to 17000 . For a $50 \mathrm{~nm}$ diameter particle placed centrally on top of the membrane of an L3 cavity (as depicted in Fig. 3) with mode volume $V=0.82(\lambda / n)^{3}$, the mode intensity at the center of the particle is $I=0.065 \cdot I_{\max }$ and $Q$ is reduced from 73000 to 66000 . Therefore, the Purcell factor $F_{p} \propto \frac{I}{I_{\max }} \frac{Q}{V}$ is larger for the $\mathrm{S} 1$ cavity by a factor

$$
\frac{F_{p}^{\mathrm{S} 1}}{F_{p}^{\mathrm{L} 3}}=\frac{0.58 \cdot \frac{17000}{0.54}}{0.065 \cdot \frac{66000}{0.82}} \cong 3.5
$$

For nanoparticles $100 \mathrm{~nm}$ in diameter, the Purcell factor for the $\mathrm{S} 1$ cavity is $\sim 9$ times higher than for the L3 cavity.

(a)
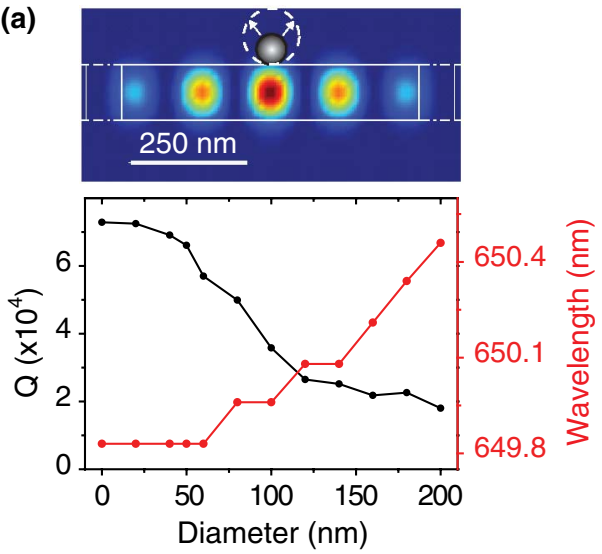

(b)
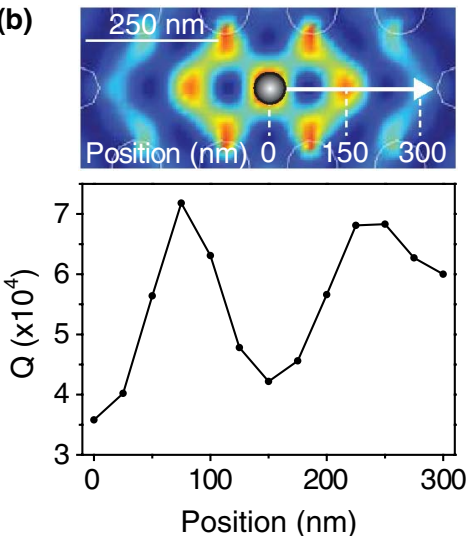

Fig. 3. (Color online) (a) Simulation of the effect of the size of a spherical nanoparticle placed centrally on top of the membrane of an L3 cavity. The original structure (hole radius $r=62 \mathrm{~nm}$, lattice constant $a=180 \mathrm{~nm}$, membrane thickness $d=120 \mathrm{~nm}$, with nearby holes modified to improve $Q$ ) has a fundamental mode with wavelength $649.83 \mathrm{~nm}$, mode volume $0.82(\lambda / n)^{3}$, and $Q_{0}=7.29 \cdot 10^{4}$. The color plot shows the size change of the nanoparticle. (b) Simulation of the effect of the position of a nanoparticle on the L3 cavity quality factor. A spherical nanoparticle (100 nm diameter) sitting on top of the membrane is laterally translated as shown in the color plot.

\section{MEASUREMENTS OF THE EFFECT OF A NANOCRYSTAL ON THE QUALITY FACTOR OF PC S1 CAVITIES}

\section{A. Cavity Fabrication}

Two types of cavities were fabricated, L3 cavities (Fig. 1(a)) and S1 cavities (Fig. 1(b)), both with and without an embedded quantum well (QW) emitting layer. We used the L3 cavities only to develop our cavity characterization method, as described below. Samples were grown by molecular beam epitaxy on a (100) GaP wafer, with a $1 \mu \mathrm{m}$ sacrificial layer of $\mathrm{Al}_{0.75} \mathrm{Ga}_{0.25} \mathrm{P}$, and a GaP membrane layer. The membrane layer in one sample was a bare $120 \mathrm{~nm}$ GaP membrane layer. In the other sample, first $55 \mathrm{~nm}$ of GaP was grown, followed by a few nanometer InGaP and again $55 \mathrm{~nm}$ of GaP, to form a membrane with an embedded QW emitting layer. PC structures were defined with electron beam lithography, after which the resist pattern was transferred into the substrate by inductively coupled plasma etching. Finally, a hydrofluoric acid (HF) chemical wet etch undercuts the sacrificial layer, leaving a freely suspended membrane.

\section{B. Cavity Characterization and Tuning}

Characterization of cavities with a QW emitting layer is done by measuring the photoluminescence (PL) spectrum in a confocal microscope. Broadband QW emission excited by $532 \mathrm{~nm}$ laser light couples to the various cavity resonances (Fig. 4(a)). The lowest energy mode of our cavities generally has the highest quality factor, up to a spectrometer-limited value of 16000 for L3 cavities and up to 3800 for S1 cavities.

While PC cavities with an embedded QW layer are straightforwardly characterized by PL measurements, they are not well suited for coupling to single quantum emitters, because the strong QW fluorescence would obscure any single photon fluorescence. Cavities with QW were therefore used only to

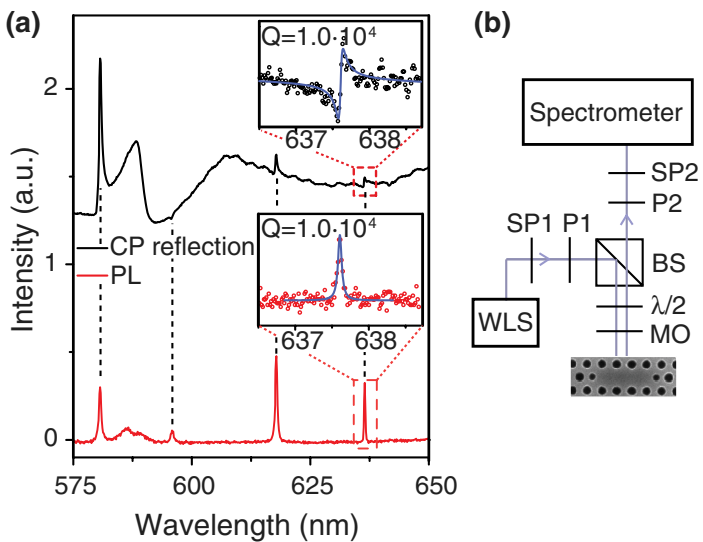

Fig. 4. (Color online) (a) Detection of L3 cavity resonances by photoluminescence (PL) and by white light crossed polarizer (CP) reflection at room temperature. In CP reflection measurements, cavity resonances have a Fano line shape, due to interference between light that is directly reflected off the surface of the membrane and the light that is scattered to the detector through the cavity [13]. Insets show a zoom of the lowest energy mode, with quality factor obtained from fitting the PL (CP reflection) data with a Lorentzian (Fano) line shape. (b) Schematic of the setup used for CP reflection detection of cavity resonances. WLS, white light source; SP, spatial filter; P, polarizer; $\lambda / 2$, half-wave plate; $\mathrm{MO}$, microscope objective $(\mathrm{NA}=0.95)$. $\mathrm{P} 1$ and P2 are oriented perpendicular to each other. The half-wave plate is used to optimize signal visibility on the spectrometer. 
tune the fabrication parameters to yield resonances around $637 \mathrm{~nm}$, which are suitable for coupling to NV centers.

Cavity resonances of samples without embedded QW can be detected using a white light crossed polarizer $(\mathrm{CP})$ reflection technique [13] (Fig. 4(b)). By comparison to a PL measurement on the same cavity (with a $\mathrm{QW}$ ), we confirm that $\mathrm{CP}$ reflection yields the same quality factors and spectral positions of the resonances (Fig. 4(a)). Cavity resonance frequencies also agree to within 1-2\% with FDTD simulations. After fabrication, the cavity resonances can be shifted using a laser-induced tuning technique [요실. By focusing a $\sim 3 \mathrm{~mW} 532 \mathrm{~nm}$ laser onto or within a few micrometers of the center of the cavity, the resonance shifts on a timescale of minutes (Figs. 5(a) and 5(b)), making it possible to tune it to within a fraction of the linewidth ( $\ll 0.1 \mathrm{~nm}$ for the data shown). The quality factor generally decreases (Fig. 5(c)), although for one cavity we observed an increase (Fig. 5(d)). We presume the increase in the cavity quality factor could be due to burning off some dirt (e.g., resist residue) with the laser. The laser-induced tuning mechanism of PC cavities is thought to originate from local oxidation of the substrate material [14].

\section{Measurement Results}

A systematic experimental study of the effect of a nanocrystal on high quality $\mathrm{PC}$ cavities requires a deterministic positioning method with nanometer accuracy. Here, we experimentally study the effect of a nanocrystal on cavity quality factors by positioning six different nanocrystals (size $\sim 50 \mathrm{~nm}$ ) into various holes of PC S1 cavities (Fig. 6 ). The positioning was done under real-time scanning electron microscope (SEM) imaging using our recently developed nanomanipulation technique $[15,16]$.

In Table 1 , we compare the cavity quality factors before and after the introduction of the nanocrystals (cavities S1-1 to S1-
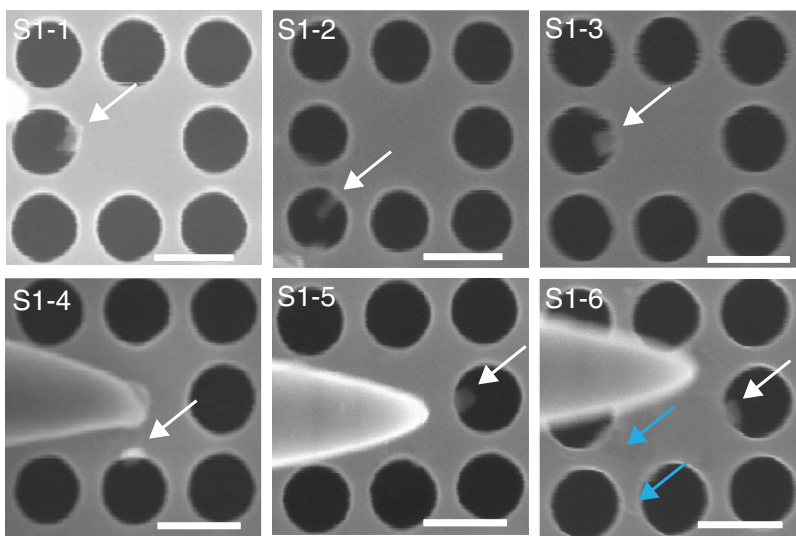

Fig. 6. (Color online) SEM images of six different diamond nanocrystals positioned into six different S1 cavities. Cavity quality factors were measured before and after the positioning to determine the effect of the nanocrystal. The results are presented in Table 1 . The white arrows indicate the position of the nanocrystal; the blue arrows indicate scratches made during positioning. Scale bars: $200 \mathrm{~nm}$.

6). We find a small decrease: $Q_{\text {after }} / Q_{\text {before }}=0.9 \pm 0.1$ from their original $Q_{\text {before }}$ of order $10^{3}$. We note that cavity S1-6 was lightly scratched with the tip during the positioning procedure (see Fig. 6), which may also have contributed to a decreased $Q$. Furthermore we note that the quality factors of the cavities used for these experiments are about an order of magnitude lower than their simulated values. We presume that a combination of fabrication imperfections and absorption losses in the GaP reduces the quality factors of the experimentally realized cavities below their simulated values.

To unambiguously attribute any measured changes of the quality factors of cavities S1-1 to S1-6 to the introduction of nanocrystals, and exclude the possibility of quality factor changes due to, e.g., manual handling of the chip, we performed a (a)

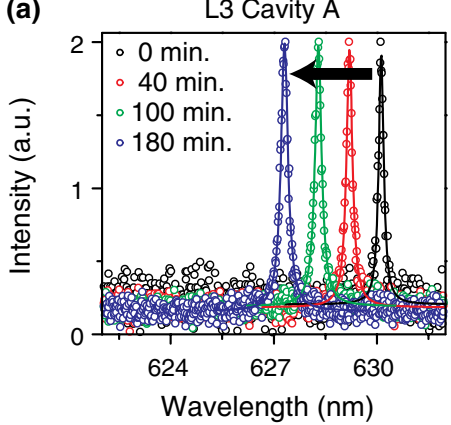

(c)

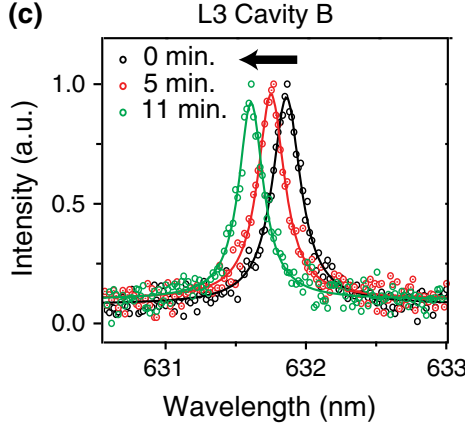

(b)
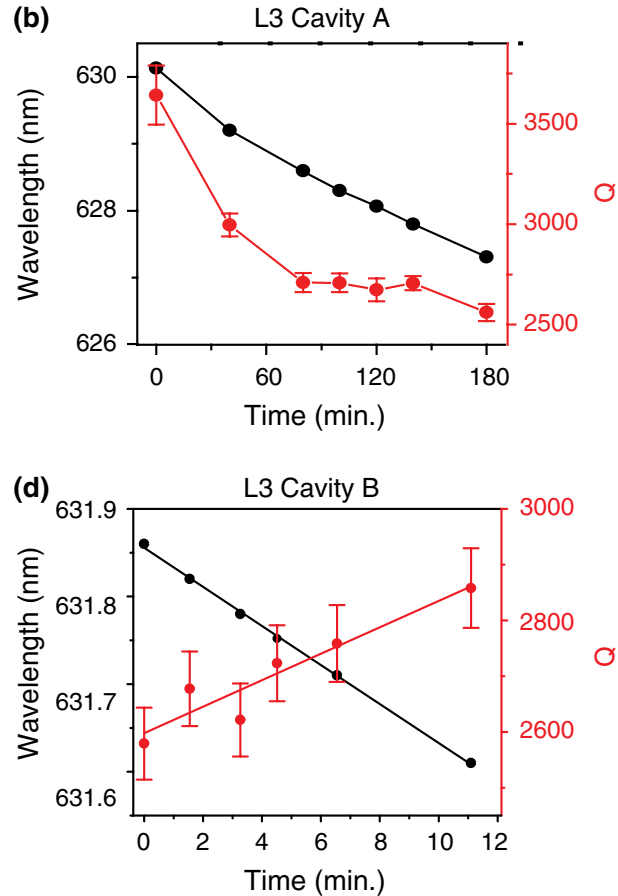

Fig. 5. (Color online) (a) Laser-induced blue shifting of the cavity resonance of L3 cavity A. The shift of the resonance wavelength and the quality factor are plotted in (b) as a function of time. (c) Laser-induced blue shifting of the cavity resonance of L3 cavity B. The shift of the resonance wavelength and the quality factor are plotted in (d) as a function of time. 
Table 1. Effect of a Nanocrystal on the Cavity Quality Factor ${ }^{a}$

\begin{tabular}{|c|c|c|c|c|c|c|c|}
\hline \multicolumn{4}{|c|}{ With nanocrystal } & \multicolumn{4}{|c|}{ Without nanocrystal } \\
\hline Cavity number & $Q\left(\cdot 10^{3}\right)$ before & $Q\left(\cdot 10^{3}\right)$ after & Changed by factor & Cavity number & $Q\left(\cdot 10^{3}\right)$ before & $Q\left(\cdot 10^{3}\right)$ after & Changed by factor \\
\hline S1-1 & $1.2(1)$ & $0.91(1)$ & $0.76(6)$ & S1-7 & $1.18(2)$ & $1.15(5)$ & $0.97(5)$ \\
\hline S1-2 & $2.2(3)$ & $1.75(6)$ & $0.8(1)$ & S1-8 & $1.60(4)$ & $1.49(1)$ & $0.93(2)$ \\
\hline S1-3 & $1.87(3)$ & $1.77(1)$ & $0.95(2)$ & S1-9 & $1.92(1)$ & $1.93(9)$ & $1.01(5)$ \\
\hline S1-4 & $1.25(2)$ & $1.30(3)$ & $1.06(3)$ & $\mathrm{S} 1-10$ & $1.7(2)$ & $1.55(4)$ & $0.9(1)$ \\
\hline S1-5 & $1.55(2)$ & $1.60(3)$ & $1.03(2)$ & \multicolumn{3}{|c|}{ Average change: } & $1.0(1)$ \\
\hline 5-8 S1-6 & $1.93(9)$ & $1.27(1)$ & $0.66(3)$ & & & & \\
\hline & Average change: & & $0.9(1)$ & & & & \\
\hline
\end{tabular}

We have measured the quality factor of six S1 cavities (S1-1 to S1-6) before and after the introduction of nanocrystals into the cavities, as shown in Fig. 6. On average, the quality factor decreased by a factor of $0.9 \pm 0.1$. Quality factors of four S1 control cavities (S1-7 to S1-10) into which we did not position a nanocrystal (Iocated on the same chip) did not decrease significantly. Numbers between parentheses denote uncertainty in the last digit, obtained from fitting CP reflection measurements of cavity resonances with a Fano lineshape.

control measurement. This control measurement showed that quality factors of cavities S1-7 to S1-10 that were located on the same chip but into which we did not position nanocrystals did not change significantly (Table 1 ). Because all the cavities S1-1 to S1-10 were located on the same chip, they simultaneously went through the same handling procedures: mounting the chip into the optical setup to measure the original quality factors of all the cavities, mounting the chip into the nanomanipulator to position nanocrystals into cavities S1-1 to S1-6, and mounting the chip into the optical setup again to measure the final quality factors of all the cavities.

To compare our measurements with theory, we now calculate the change in quality factor that is expected from simulations:

$$
\frac{1}{Q_{\text {after }}}=\frac{1}{Q_{\text {before }}}+\frac{1}{Q_{\text {scattering }}},
$$

where $Q_{\text {after }}$ is the quality factor we expect after a nanocrystal is introduced into a cavity with original quality factor $Q_{\text {before }}$. The effect of scattering off the nanocrystal is contained in $Q_{\text {scattering, }}$, which can be calculated from our simulations

$$
\frac{1}{Q_{\text {scattering }}}=\frac{1}{Q_{\text {after }}^{\text {sim }}}-\frac{1}{Q_{\text {before }}^{\text {sim }}},
$$

where $Q_{\text {before }}^{\text {sim }}\left(Q_{\text {after }}^{\text {sim }}\right)$ is the simulated quality factor before (after) the introduction of a nanocrystal (see Fig. 2). Using Eqs. (2) and (3) and the results from Section $2 \underline{\text {, we obtain }}$ $Q_{\text {after }} \approx 0.99 Q_{\text {before }}$ for a $50 \mathrm{~nm}$ nanocrystal placed at the center of the membrane in the nearest airhole of an S1 cavity having $Q_{\text {before }}=1500$.

We conclude that only a slight reduction in quality factor is expected from simulations, since we have positioned nanocrystals into cavities with quality factors that, even without a nanocrystal, were an order of magnitude lower than the quality factors obtained in simulations of cavities with a nanocrystal. Our measurements confirm that a nanoparticle has just a minor effect on cavities with $Q$ of order $10^{3}$, a promising result for future work on cavity-QED systems in the solid state and with diamond defect centers in particular.

To illustrate why this is a promising result, we now compute the maximum attainable Purcell factor for an emitter when its $50 \mathrm{~nm}$ host nanoparticle is placed in the middle of the membrane against the inner edge of a hole of an S1 cavity having $Q=1500$ and $V=0.56\left(\lambda_{\text {cav }} / n\right)^{3}$ (as in Fig. 2):

$$
F_{P}=0.58 \frac{3 Q}{4 \pi^{2} V\left(\lambda_{\text {cav }} / n\right)^{3}}=118
$$

where we have assumed that the emitter is at the center of the particle (where $I=0.58 I_{\max }$ ) and that the dipole moment of the emitter is aligned with the polarization of the mode.

The low coherent photon emission rate of an (uncoupled) $\mathrm{NV}$ center is the major hurdle for its application in many quantum optical experiments, such as for measurement-based entanglement of distant NV centers (see, e.g., Barrett and Kok [17]). Our results show that the coherent photon emission rate can in principle be enhanced by a factor of 118 by coupling an $\mathrm{NV}$ center embedded in a $50 \mathrm{~nm}$ diamond nanocrystal to an S1 cavity. This would dramatically reduce the measurement time required to demonstrate spin-spin entanglement between two such coupled NV center-PC cavity systems by a factor of $118^{2}$. We note that this does not require strong coupling between cavity and NV center, as it exploits the Purcell effect in the weak coupling-bad cavity regime. For the type of PC cavities used in the experiments in this article, a $Q$ of order $10^{5}$ is required to reach the strong-coupling regime.

\section{CONCLUSIONS}

In conclusion, we have described the fabrication, characterization, and tuning of high quality GaP PC cavities that resonate in the visible. We have studied the effect of positioning diamond nanocrystals into the confined optical mode on the optical properties of both $\mathrm{S} 1$ and L3 GaP cavities by simulations and by measurements.

From simulations, we find that quality factors remain above $\sim 10^{4}$ for diamond particles up to $100 \mathrm{~nm}$ in diameter for both types of cavities. Furthermore, our simulations show that the maximum attainable coupling for an emitter inside a $50 \mathrm{~nm}$ $(100 \mathrm{~nm})$ nanoparticle placed into a hole of an S1 cavity is a factor of $\sim 3.5(9)$ higher than when a particle of the same size is placed centrally on top of the membrane of an L3 cavity. We therefore conclude that our simulations indicate that the $\mathrm{S} 1$ cavity is a better candidate for coupling to external emitters than the more commonly used L3 cavity.

Experimentally, we have compared quality factors of six PC S1 cavities before and after positioning a nanocrystal into the cavity. We find only a slight decrease by a factor of $0.9 \pm 0.1$ on average.

\section{ACKNOWLEDGMENTS}

This work is supported by stichting voor Fundamenteel Onderzoek der Materie (FOM) and the Nederlandse 
Organisatie voor Wetenschappelijk Onderzoek (NWO). T. H. O. acknowledges support from Technologiestichting STW and from an ERC Starting Grant. D. B. acknowledges support from NSF grant 0901886 and Marie Curie EXT-CT-2006042580. J. H. acknowledges support from a U.S. Department of Education GAANN grant.

\section{REFERENCES}

1. R. Loudon, The Quantum Theory of Light (Oxford University, 2000).

2. S. Noda, M. Fujita, and T. Asano, "Spontaneous-emission control by photonic crystals and nanocavities," Nature Photon. 1, 449-458 (2007).

3. C. Santori, D. Fattal, J. Vučković, G. S. Solomon, and Y. Yamamoto, "Indistinguishable photons from a single-photon device," Nature 419, 594-597 (2002).

4. J. P. Reithmaier, G. Sek, A. Löffler, C. Hofmann, S. Kuhn, S Reitzenstein, L. V. Keldysh, V. D. Kulakovskii, T. L. Reinecke, and A. Forchel, "Strong coupling in a single quantum dotsemiconductor microcavity system," Nature 432, 197-200 (2004).

5. A. Badolato, K. Hennessy, M. Atatüre, J. Dreiser, E. Hu, P. M Petroff, and A. Imamoğlu, "Deterministic coupling of single quantum dots to single nanocavity modes," Science $\mathbf{3 0 8}$, 1158-1161 (2005).

6. S. M. Thon, M. T. Rakher, H. Kim, J. Gudat, W. T. M. Irvine, P. M. Petroff, and D. Bouwmeester, "Strong coupling through optical positioning of a quantum dot in a photonic crystal cavity," Appl. Phys. Lett. 94, 111115 (2009).

7. K. Rivoire, A. Kinkhabwala, F. Hatami, W. T. Masselink, Y. Avlasevich, K. Müllen, W. E. Moerner, and J. Vučković, "Lithographic positioning of fluorescent molecules on high- $Q$ photonic crystal cavities," Appl. Phys. Lett. 95, 123113 (2009).

8. J. Wolters, A. W. Schell, G. Kewes, N. Nüsse, M. Schoengen, H. Döscher, T. Hannappel, B. Löchel, M. Barth, and O. Benson, "En- hancement of the zero phonon line emission from a single nitrogen vacancy center in a nanodiamond via coupling to a photonic crystal cavity," Appl. Phys. Lett. 97, 141108 (2010).

9. M. Barth, N. Nüsse, B. Löchel, and O. Benson, "Controlled coupling of a single-diamond nanocrystal to a photonic crystal cavity," Opt. Lett. 34, 1108-1110 (2009).

10. D. Englund, B. Shields, K. Rivoire, F. Hatami, J. Vučković, H Park, and M. D. Lukin, "Deterministic coupling of a single nitrogen vacancy center to a photonic crystal cavity," Nano Lett. 10 3922-3926 (2010).

11. T. van der Sar, J. Hagemeier, W. Pfaff, E. C. Heeres, T. H. Oosterkamp, D. Bouwmeester, and R. Hanson, "Deterministic nano-assembly of a coupled quantum emitter-photonic crystal cavity system," Appl. Phys. Lett. 98, 193103 (2011).

12. A. F. Koenderink, M. Kafesaki, B. Buchler, and V. Sandoghdar, "Controlling the resonance of a photonic crystal microcavity by a near-field probe,” Phys. Rev. Lett. 95, 153904 (2005).

13. M. Galli, S. L. Portalupi, M. Belotti, L. C. Andreani, L. O'Faolain, and T. F. Krauss, "Light scattering and Fano resonances in high$Q$ photonic crystal nanocavities," Appl. Phys. Lett. 94, 071101 (2009).

14. H. S. Lee, S. Kiravittaya, S. Kumar, J. D. Plumhof, L. Balet, L. H Li, M. Francardi, A. Gerardino, A. Fiore, A. Rastelli, and O. G. Schmidt, "Local tuning of photonic crystal nanocavity modes by laser-assisted oxidation," Appl. Phys. Lett. 95, 191109 (2009).

15. E. C. Heeres, A. J. Katan, M. H. van Es, A. F. Beker, M. Hesselberth, D. J. van Der Zalm, and T. H. Oosterkamp, "A compact multipurpose nanomanipulator for use inside a scanning electron microscope," Rev. Sci. Instrum. 81, 023704 (2010).

16. T. van der Sar, E. C. Heeres, G. M. Dmochowski, G. de Lange, L. Robledo, T. H. Oosterkamp, and R. Hanson, "Nanopositioning of a diamond nanocrystal containing a single nitrogen-vacancy defect center," Appl. Phys. Lett. 94, 173104 (2009).

17. S. D. Barrett and P. Kok, "Efficient high-fidelity quantum computation using matter qubits and linear optical," Phys. Rev. Lett. 71, 060310 (2005). 